STOCHASTIC MODELING AND CONTROL

BANACH CENTER PUBLICATIONS, VOLUME 122

INSTITUTE OF MATHEMATICS

POLISH ACADEMY OF SCIENCES

WARSZAWA 2020

\title{
PAIRS TRADING: AN OPTIMAL SELLING RULE UNDER A REGIME SWITCHING MODEL
}

\author{
JINGZHI TIE and QING ZHANG \\ Department of Mathematics, University of Georgia \\ Athens, GA 30602, USA \\ ORCID: 0000-0001-6212-7056, 0000-0002-8846-5587 \\ E-mail: jtie@uga.edu,qz@uga.edu
}

\begin{abstract}
This paper is concerned with an optimal selling rule for pairs stock trading. A pairs position consists of a long position in one stock and a short position in the other. The problem is to find an optimal stopping time to close the pairs position by selling the long position and buying back the short position. In this paper, we consider the optimal pairs-trading selling rule by allowing the stock prices to follow a general geometric Brownian motion with regime switching. The optimal policy is characterized by threshold curves obtained by solving the associated HJB equations (quasi-variational inequalities). Moreover, numerical examples are provided to illustrate optimal policies and value functions.
\end{abstract}

1. Introduction. Pairs trading is about simultaneously trading of a pair of stocks. A pairs position consists of a long position in one stock and a short position in the other. In this paper, assuming an existing pairs position, our goal is to determine when to fold and close the position.

Pairs trading is closely related to the timing of the optimal investments studied in McDonald and Siegel [MS. In particular, they considered the optimal timing of investment in an irreversible project. Two main variables in their model are the value of the project and the cost of investing. They demonstrated one should defer the investment until the present value of the benefits from the project exceed the investment cost by a certain margin. Further studies along this line were carried out by $\mathrm{Hu}$ and Øksendal [HO] to specify precise optimality conditions and to provide a new proof of the following

2010 Mathematics Subject Classification: Primary 91G80; Secondary 93E20.

Key words and phrases: pairs trading, optimal policy, quasi-variational inequalities, regime switching

The paper is in final form and no version of it will be published elsewhere. 
variational inequalities among others. Their results can be easily interpreted in terms of pairs-trade selling rule when treating the project value as the long position and investment cost the short position.

In this paper, we extend these results to incorporate markets with regime switching. We focus on a simple and easily implementable strategy, and its optimality and sufficient conditions for a closed-form solution.

Mathematical trading rules have been studied for many years. For example, Zhang [Z] considered a selling rule determined by two threshold levels, a target price, and a stoploss limit. In [Z], such optimal threshold levels are obtained by solving a set of two-point boundary value problems. Guo and Zhang [GZ] studied the optimal selling rule under a model with switching Geometric Brownian motion. Using a smooth-fit technique, they obtained the optimal threshold levels by solving a set of algebraic equations. These papers are concerned with the selling side of trading in which the underlying price models are of GBM type. Recently, Dai et al. DZZ developed a trend-following rule based on a conditional probability indicator. They showed that the optimal trading rule can be determined by two threshold curves which can be obtained by solving the associated Hamilton-Jacobi-Bellman (HJB) equations. A similar idea was developed following a confidence interval approach by Iwarere and Barmish [IB. Besides, Merhi and Zervos [MZ] studied an investment capacity expansion/reduction problem following a dynamic programming approach under a geometric Brownian motion market model. In connection with mean reversion trading, Zhang and Zhang [ZZ] obtained a buy-low and sell-high policy by characterizing the 'low' and 'high' levels in terms of the mean reversion parameters. Song and Zhang [SZ studied pairs trading under a mean reversion model. It is shown that the optimal trading rule can be determined by threshold levels that can be obtained by solving a set of algebraic equations. A set of sufficient conditions are also provided to establish the desired optimality. Deshpande and Barmish [DB] introduced a controltheoretic approach. In particular, they were able to relax the requirement for spread functions and showed that their trading algorithm produces positive expected returns. Other related pairs technologies can be found in Elliott et al. [EHM] and Whistler [W]. Recently, we TZZ studied an optimal pairs trading rule. The objective is to initiate and close the positions of the pair sequentially to maximize a discounted payoff function. Using a dynamic programming approach, we study the problem under a geometric Brownian motion model and proved that the buying and selling can be determined by two threshold curves in closed form. They also demonstrate the optimality of their trading strategy.

Market models with regime switching are important in market analysis. In this paper, we consider a geometric Brownian motion with regime switching. The market mode is represented by a two-state Markov chain. We focus on the selling part of pairs trading and generalize the results of $\mathrm{Hu}$ and $\varnothing \mathrm{ksendal}[\mathrm{HO}$ by incorporating models with regime switching. We show that the optimal selling rule can be determined by two threshold curves and establish a set of sufficient conditions that guarantee the optimality of the policy. We also include several numerical examples under a different set of parameter values. 
This paper is organized as follows. In §2, we formulate the pairs trading problem under consideration. In $\S 3$, we study the associated HJB equations and their solutions. We provide a set of sufficient conditions that guarantee the optimality of our selling rule. Numerical examples are given in $\S 4$. Some concluding remarks are given in $\S 5$.

2. Problem formulation. We consider two stocks $\mathbf{S}^{1}$ and $\mathbf{S}^{2}$. Let $\left\{X_{t}^{1}, t \geq 0\right\}$ denote the prices of stock $\mathbf{S}^{1}$ and $\left\{X_{t}^{2}, t \geq 0\right\}$ that of stock $\mathbf{S}^{2}$. They satisfy the following stochastic differential equation:

$$
d\left(\begin{array}{c}
X_{t}^{1} \\
X_{t}^{2}
\end{array}\right)=\left(\begin{array}{cc}
X_{t}^{1} & \\
& X_{t}^{2}
\end{array}\right)\left[\left(\begin{array}{c}
\mu_{1}\left(\alpha_{t}\right) \\
\mu_{2}\left(\alpha_{t}\right)
\end{array}\right) d t+\left(\begin{array}{ll}
\sigma_{11}\left(\alpha_{t}\right) & \sigma_{12}\left(\alpha_{t}\right) \\
\sigma_{21}\left(\alpha_{t}\right) & \sigma_{22}\left(\alpha_{t}\right)
\end{array}\right) d\left(\begin{array}{c}
W_{t}^{1} \\
W_{t}^{2}
\end{array}\right)\right],
$$

where $\mu_{i}, i=1,2$, are the return rates, $\sigma_{i j}, i, j=1,2$, the volatility constants, $\alpha_{t}$ a two-state Markov chain, and $\left(W_{t}^{1}, W_{t}^{2}\right)$ a two-dimensional standard Brownian motion.

Let $\mathcal{M}=\{1,2\}$ denote the state space for the Markov chain $\alpha_{t}$ and let $Q=$ $\left(\begin{array}{cc}-\lambda_{1} & \lambda_{1} \\ \lambda_{2} & -\lambda_{2}\end{array}\right)$, with $\lambda_{1}>0$ and $\lambda_{2}>0$, be its generator. We assume $\alpha_{t}$ and $\left(W_{t}^{1}, W_{t}^{2}\right)$ are independent.

In this paper, we consider a pair selling rule. For simplicity, we assume the corresponding pair's position consists of a one-share long position in stock $\mathbf{S}^{1}$ and a one-share short position in stock $\mathbf{S}^{2}$. The problem is to determine an optimal stopping time $\tau$ to close the pair's position by selling $\mathbf{S}^{1}$ and buying back $\mathbf{S}^{2}$.

Let $K$ denote the transaction cost percentage (e.g., slippage and/or commission) associated with stock transactions. For example, the proceeds to close the pairs position at $t$ is $(1-K) X_{t}^{1}-(1+K) X_{t}^{2}$. For ease of notation, let $\beta_{\mathrm{b}}=1+K$ and $\beta_{\mathrm{s}}=1-K$.

Given the initial state $\left(x_{1}, x_{2}\right), \alpha=1,2$, and the selling time $\tau$, the corresponding reward function

$$
J\left(x_{1}, x_{2}, \alpha, \tau\right)=E\left[e^{-\rho \tau}\left(\beta_{\mathrm{s}} X_{\tau}^{1}-\beta_{\mathrm{b}} X_{\tau}^{2}\right)\right],
$$

where $\rho>0$ is a given discount factor.

Let $\mathcal{F}_{t}=\sigma\left\{\left(X_{r}^{1}, X_{r}^{2}, \alpha_{r}\right): r \leq t\right\}$. The problem is to find an $\left\{\mathcal{F}_{t}\right\}$ stopping time $\tau$ to maximize $J$. Let $V\left(x_{1}, x_{2}, \alpha\right)$ denote the corresponding value functions:

$$
V\left(x_{1}, x_{2}, \alpha\right)=\sup _{\tau} J\left(x_{1}, x_{2}, \alpha, \tau\right) .
$$

REMARK 2.1. The 'one-share' pair position is not as restrictive as it appears. For example, one can consider any pairs with $n_{1}$ shares of long position in $\mathbf{S}^{1}$ and $n_{2}$ shares of short position in $\mathbf{S}^{2}$. To treat this case, one only has to make change of the state variables $\left(X_{t}^{1}, X_{t}^{2}\right) \rightarrow\left(n_{1} X_{t}^{1}, n_{2} X_{t}^{2}\right)$. Due to the nature of GBMs, the corresponding system equation in (1) will remain the same. The modification only affects the reward function in 2 implicitly.

Throughout this paper, we impose the following conditions:

(A1) For $\alpha=1,2, \rho>\mu_{1}(\alpha)$ and $\rho>\mu_{2}(\alpha)$.

Under these conditions, we have the lower and upper bounds for $V$ :

$$
\beta_{\mathrm{s}} x_{1}-\beta_{\mathrm{b}} x_{2} \leq V\left(x_{1}, x_{2}, \alpha\right) \leq \beta_{\mathrm{s}} x_{1} .
$$


Actually, the lower bound follows from the value function definition

$$
V\left(x_{1}, x_{2}, \alpha\right) \geq J\left(x_{1}, x_{2}, \alpha, 0\right)=\beta_{\mathrm{s}} x_{1}-\beta_{\mathrm{b}} x_{2} .
$$

The upper bound can be obtained from Dynkin's formula

$$
J\left(x_{1}, x_{2}, \alpha, \tau\right) \leq E\left[e^{-\rho \tau} \beta_{\mathrm{s}} X_{\tau}^{1}\right]=\beta_{\mathrm{s}}\left(x_{1}+E \int_{0}^{\tau} e^{-\rho t} X_{t}^{1}\left(-\rho+\mu_{1}\left(\alpha_{t}\right)\right) d t\right) \leq \beta_{\mathrm{s}} x_{1} .
$$

3. HJB equations. In this paper, we follow the dynamic programming approach and focus on HJB equations. First, for $i=1,2$, let

$$
\begin{aligned}
\mathcal{A}_{i}=\frac{1}{2}\left[a_{11}(i) x_{1}^{2} \frac{\partial^{2}}{\partial x_{1}^{2}}+2 a_{12}(i) x_{1} x_{2} \frac{\partial^{2}}{\partial x_{1} \partial x_{2}}+a_{22}(i) x_{2}^{2} \frac{\partial^{2}}{\partial x_{2}^{2}}\right] & \\
& +\mu_{1}(i) x_{1} \frac{\partial}{\partial x_{1}}+\mu_{2}(i) x_{2} \frac{\partial}{\partial x_{2}}
\end{aligned}
$$

where

$a_{11}(i)=\sigma_{11}^{2}(i)+\sigma_{12}^{2}(i), a_{12}(i)=\sigma_{11}(i) \sigma_{21}(i)+\sigma_{12}(i) \sigma_{22}(i)$, and $a_{22}(i)=\sigma_{21}^{2}(i)+\sigma_{22}^{2}(i)$.

Formally, the associated HJB equations have the form:

$$
\left\{\begin{array}{r}
\min \left\{\left(\rho-\mathcal{A}_{1}\right) v\left(x_{1}, x_{2}, 1\right)-\lambda_{1}\left(v\left(x_{1}, x_{2}, 2\right)-v\left(x_{1}, x_{2}, 1\right)\right),\right. \\
\left.v\left(x_{1}, x_{2}, 1\right)-\beta_{\mathrm{s}} x_{1}+\beta_{\mathrm{b}} x_{2}\right\}=0, \\
\min \left\{\left(\rho-\mathcal{A}_{2}\right) v\left(x_{1}, x_{2}, 2\right)-\lambda_{2}\left(v\left(x_{1}, x_{2}, 1\right)-v\left(x_{1}, x_{2}, 2\right)\right),\right. \\
\left.v\left(x_{1}, x_{2}, 2\right)-\beta_{\mathrm{s}} x_{1}+\beta_{\mathrm{b}} x_{2}\right\}=0 .
\end{array}\right.
$$

To solve the HJB equations, we first convert them into equations with a single independent variable by introducing $y=x_{2} / x_{1}$ and $v\left(x_{1}, x_{2}, i\right)=x_{1} w_{i}\left(x_{2} / x_{1}\right)$, for some function $w_{i}(y)$ and $i=1,2$. Then direct calculation yields

$$
\begin{aligned}
& \frac{\partial v\left(x_{1}, x_{2}, i\right)}{\partial x_{1}}=w_{i}(y)-y w_{i}^{\prime}(y), \quad \frac{\partial v\left(x_{1}, x_{2}, i\right)}{\partial x_{2}}=w_{i}^{\prime}(y), \\
& \frac{\partial^{2} v\left(x_{1}, x_{2}, i\right)}{\partial x_{1}^{2}}=\frac{y^{2} w_{i}^{\prime \prime}(y)}{x_{1}}, \quad \frac{\partial^{2} v\left(x_{1}, x_{2}, i\right)}{\partial x_{2}^{2}}=\frac{w_{i}^{\prime \prime}(y)}{x_{1}}, \quad \frac{\partial^{2} v\left(x_{1}, x_{2}, i\right)}{\partial x_{1} \partial x_{2}}=-\frac{y w_{i}^{\prime \prime}(y)}{x_{1}} .
\end{aligned}
$$

We rewrite $\mathcal{A}_{i} v\left(x_{1}, x_{2}, i\right)$ in terms of $w_{i}$ to obtain

$$
\mathcal{A}_{i} v\left(x_{1}, x_{2}, i\right)=x_{1}\left\{\sigma_{i} y^{2} w_{i}^{\prime \prime}(y)+\left[\mu_{2}(i)-\mu_{1}(i)\right] y w_{i}^{\prime}(y)+\mu_{1}(i) w_{i}(y)\right\} .
$$

where $\sigma_{i}=\left[a_{11}(i)-2 a_{12}(i)+a_{22}(i)\right] / 2$. Let

$$
\mathcal{L}_{i}\left[w_{i}(y)\right]=\sigma_{i} y^{2} w_{i}^{\prime \prime}(y)+\left[\mu_{2}(i)-\mu_{1}(i)\right] y w_{i}^{\prime}(y)+\mu_{1}(i) w_{i}(y), \quad i=1,2 .
$$

Then, the HJB equations can be given in terms of $y$ and $w_{i}$ as follows:

$$
\left\{\begin{array}{l}
\min \left\{\left(\rho+\lambda_{1}-\mathcal{L}_{1}\right) w_{1}(y)-\lambda_{1} w_{2}(y), w_{1}(y)+\beta_{\mathrm{b}} y-\beta_{\mathrm{s}}\right\}=0, \\
\min \left\{\left(\rho+\lambda_{2}-\mathcal{L}_{2}\right) w_{2}(y)-\lambda_{2} w_{1}(y), w_{2}(y)+\beta_{\mathrm{b}} y-\beta_{\mathrm{s}}\right\}=0 .
\end{array}\right.
$$

In this paper, we only consider the case when $\sigma_{i} \neq 0, i=1,2$. If either $\sigma_{1}=0$ and/or $\sigma_{2}=0$, the problem reduces to a (partial) first order case and can be treated in a similar and much simpler way. 
First we consider the equations:

$$
\left(\rho+\lambda_{1}-\mathcal{L}_{1}\right) w_{1}=\lambda_{1} w_{2} \quad \text { and } \quad\left(\rho+\lambda_{2}-\mathcal{L}_{2}\right) w_{2}=\lambda_{2} w_{1} .
$$

Then both $w_{1}$ and $w_{2}$ satisfy the equation

$$
\left[\left(\rho+\lambda_{1}-\mathcal{L}_{1}\right)\left(\rho+\lambda_{2}-\mathcal{L}_{2}\right)-\lambda_{1} \lambda_{2}\right] w=0 .
$$

Note that both $\mathcal{L}_{1}$ and $\mathcal{L}_{2}$ are the classical Euler type operators and therefore the solutions to the above equation is of the form $w=y^{\delta}$ for some $\delta$. This leads to that $\delta$ must satisfy

$$
\left[\rho+\lambda_{1}-A_{1}(\delta)\right]\left[\rho+\lambda_{2}-A_{2}(\delta)\right]-\lambda_{1} \lambda_{2}=0
$$

with

$$
A_{i}(\delta)=\sigma_{i} \delta(\delta-1)+\left[\mu_{2}(i)-\mu_{1}(i)\right] \delta+\mu_{1}(i), \quad i=1,2 .
$$

It is elementary to show the equation (9) has four zeros which can be arranged as follows: $\delta_{1} \geq \delta_{2}>1>0>\delta_{3} \geq \delta_{4}$.

Let

$$
w_{1}=\sum_{j=1}^{4} c_{1 j} y^{\delta_{j}} \quad \text { and } \quad w_{2}=\sum_{j=1}^{4} c_{2 j} y^{\delta_{j}},
$$

for some constants $c_{i j}$. Then, in view of the equations in (8), it follows that

$$
c_{1, j}\left(\rho+\lambda_{1}-A_{1}\left(\delta_{j}\right)\right)=\lambda_{1} c_{2 j} \quad \text { and } \quad c_{2 j}\left(\rho+\lambda_{2}-A_{2}\left(\delta_{j}\right)\right)=\lambda_{2} c_{1 j} .
$$

Define

$$
\eta_{j}=\frac{\rho+\lambda_{1}-A_{1}\left(\delta_{j}\right)}{\lambda_{1}}
$$

Then it follows from (9) that

$$
\eta_{j}=\frac{\lambda_{2}}{\rho+\lambda_{2}-A_{2}\left(\delta_{j}\right)} .
$$

Therefore, $c_{2 j}=\eta_{j} c_{1 j}, j=1,2,3,4$. Hence,

$$
w_{1}=\sum_{j=1}^{4} c_{1 j} y^{\delta_{j}} \quad \text { and } \quad w_{2}=\sum_{j=1}^{4} \eta_{j} c_{1 j} y^{\delta_{j}}
$$

will be the general solution of (8).

Heuristically, one should close the pairs position when $X_{t}^{1}$ is large and $X_{t}^{2}$ is small. In view of this, we introduce $H_{1}=\left\{\left(x_{1}, x_{2}\right): x_{2} \leq k_{1} x_{1}\right\}$ and $H_{2}=\left\{\left(x_{1}, x_{2}\right): x_{2} \leq k_{2} x_{1}\right\}$, for some $k_{1}$ and $k_{2}$ so that one should sell when $\left(X_{t}^{1}, X_{t}^{2}\right)$ enters $H_{i}$ provided $\alpha_{t}=i$, $i=1,2$.

In this paper, we need consider two cases: $k_{1} \leq k_{2}$ and $k_{2} \geq k_{1}$. By symmetry in $\alpha_{t}=1$ and $\alpha_{t}=2$, we only need to consider one of them, say $k_{1} \leq k_{2}$. We treat two separate cases: $k_{1}<k_{2}$ and $k_{1}=k_{2}$.

Case 1: $k_{1}<k_{2}$. First, we divide $(0, \infty)$ into three intervals:

$$
\Gamma_{1}=\left(0, k_{1}\right], \quad \Gamma_{2}=\left(k_{1}, k_{2}\right), \quad \text { and } \quad \Gamma_{3}=\left[k_{2}, \infty\right) .
$$


Then, on each of these intervals, the HJB equations can be specified as follows:

$$
\left\{\begin{array}{lll}
\Gamma_{1}: & w_{1}(y)=\beta_{\mathrm{s}}-\beta_{\mathrm{b}} y ; & w_{2}(y)=\beta_{\mathrm{s}}-\beta_{\mathrm{b}} y \\
\Gamma_{2}: & \left(\rho+\lambda_{1}-\mathcal{L}_{1}\right) w_{1}(y)=\lambda_{1} w_{2}(y) ; & w_{2}(y)=\beta_{\mathrm{s}}-\beta_{\mathrm{b}} y \\
\Gamma_{3}: & \left(\rho+\lambda_{1}-\mathcal{L}_{1}\right) w_{1}(y)=\lambda_{1} w_{2}(y) ; & \left(\rho+\lambda_{2}-\mathcal{L}_{2}\right) w_{2}(y)=\lambda_{2} w_{1}(y) .
\end{array}\right.
$$

We are to find solutions on each intervals. First, on $\Gamma_{3}$, recall the linear bounds for value functions given in (4). Recall also that $\delta_{1}>1$ and $\delta_{2}>1$. It follows that the coefficients in 12 for $y^{\delta_{1}}$ and $y^{\delta_{2}}$ must be zero. Therefore,

$$
w_{1}=C_{1} y^{\delta_{3}}+C_{2} y^{\delta_{4}} \text { and } w_{2}=\eta_{3} C_{1} y^{\delta_{3}}+\eta_{4} C_{2} y^{\delta_{4}} .
$$

Next, to find solution on $\Gamma_{2}$, note that a particular solution for

$$
\left(\rho+\lambda_{1}-\mathcal{L}_{1}\right) w_{1}(y)=\lambda_{1} w_{2}(y)=\lambda_{1}\left(\beta_{\mathrm{s}}-\beta_{\mathrm{b}} y\right)
$$

can be given by $w_{1}=a_{1}+a_{2} y$, with

$$
a_{1}=\frac{\lambda_{1} \beta_{\mathrm{s}}}{\rho+\lambda_{1}-\mu_{1}(1)} \quad \text { and } \quad a_{2}=-\frac{\lambda_{1} \beta_{\mathrm{b}}}{\rho+\lambda_{1}-\mu_{2}(1)} .
$$

To find a general solution of the above non-homogeneous equation, we only need to solve the homogeneous equation $\left(\rho+\lambda_{1}-\mathcal{L}_{1}\right) w_{1}=0$. This is also of Euler type and its solution is of the form $y^{\gamma}$. Then $\gamma$ must be the roots of the quadratic equation

$$
\sigma_{1} \gamma(\gamma-1)+\left[\mu_{2}(1)-\mu_{1}(1)\right] \gamma+\mu_{1}(1)-\rho-\lambda_{1}=0 .
$$

They are given by

$$
\left\{\begin{array}{l}
\gamma_{1}=\frac{1}{2}+\frac{\mu_{1}(1)-\mu_{2}(1)}{2 \sigma_{1}}+\sqrt{\left(\frac{1}{2}+\frac{\mu_{1}(1)-\mu_{2}(1)}{2 \sigma_{1}}\right)^{2}+\frac{\rho+\lambda_{1}-\mu_{1}(1)}{\sigma_{1}}} \\
\gamma_{2}=\frac{1}{2}+\frac{\mu_{1}(1)-\mu_{2}(1)}{2 \sigma_{1}}-\sqrt{\left(\frac{1}{2}+\frac{\mu_{1}(1)-\mu_{2}(1)}{2 \sigma_{1}}\right)^{2}+\frac{\rho+\lambda_{1}-\mu_{1}(1)}{\sigma_{1}}} .
\end{array}\right.
$$

The general solution for $w_{1}$ on $\Gamma_{2}$ is given by

$$
w_{1}=C_{3} y^{\gamma_{1}}+C_{4} y^{\gamma_{2}}+\frac{\lambda_{1} \beta_{\mathrm{s}}}{\rho+\lambda_{1}-\mu_{1}(1)}-\frac{\lambda_{1} \beta_{\mathrm{b}}}{\rho+\lambda_{1}-\mu_{2}(1)} y,
$$

for some constants $C_{3}$ and $C_{4}$.

Smooth-fit conditions. Smooth-fit conditions in connection with optimal stopping typically require the value functions to be continuously differentiable. Next we use such smooth-fit conditions to set up equations for parameters $C_{j}, j=1,2,3,4, k_{1}$ and $k_{2}$.

First, the continuous differentiability of $w_{1}$ at $k_{1}$ yields

$$
\begin{aligned}
\beta_{\mathrm{s}}-\beta_{\mathrm{b}} k_{1} & =C_{3} k_{1}^{\gamma_{1}}+C_{4} k_{1}^{\gamma_{2}}+a_{1}+a_{2} k_{1}, \\
-\beta_{\mathrm{b}} & =C_{3} \gamma_{1} k_{1}^{\gamma_{1}-1}+C_{4} \gamma_{2} k_{1}^{\gamma_{2}-1}+a_{2} .
\end{aligned}
$$

Similarly, we have the equation for $w_{2}$ at $k_{2}$

$$
\begin{aligned}
\beta_{\mathrm{s}}-\beta_{\mathrm{b}} k_{2} & =\eta_{3} C_{1} k_{2}^{\delta_{3}}+\eta_{4} C_{2} k_{2}^{\delta_{4}}, \\
-\beta_{\mathrm{b}} & =\eta_{3} \delta_{3} C_{1} k_{2}^{\delta_{3}-1}+\eta_{4} \delta_{4} C_{2} k_{2}^{\delta_{4}-1} .
\end{aligned}
$$


Finally, the equations for $w_{1}$ at $k_{2}$ are given by

$$
\begin{aligned}
C_{3} k_{2}^{\gamma_{1}}+C_{4} k_{2}^{\gamma_{2}}+a_{1}+a_{2} k_{2} & =C_{1} k_{2}^{\delta_{3}}+C_{2} k_{2}^{\delta_{4}}, \\
C_{3} \gamma_{1} k_{2}^{\gamma_{1}-1}+C_{4} \gamma_{2} k_{2}^{\gamma_{2}-1}+a_{2} & =\delta_{3} C_{1} k_{2}^{\delta_{3}-1}+\delta_{4} C_{2} k_{2}^{\delta_{4}-1} .
\end{aligned}
$$

We solve equations 16 and 17 for $C_{j}, j=1,2,3,4$, in terms of $k_{1}$ and $k_{2}$ and obtain

$$
\left\{\begin{array}{l}
C_{1}=\frac{-\delta_{4} \beta_{\mathrm{s}}+\left(\delta_{4}-1\right) \beta_{\mathrm{b}} k_{2}}{\eta_{3}\left(\delta_{3}-\delta_{4}\right) k_{2}^{\delta_{3}}}, \\
C_{2}=\frac{\delta_{3} \beta_{\mathrm{s}}+\left(1-\delta_{3}\right) \beta_{\mathrm{b}} k_{2}}{\eta_{4}\left(\delta_{3}-\delta_{4}\right) k_{2}^{\delta_{4}}} \\
C_{3}=\frac{\gamma_{2}\left(\beta_{\mathrm{s}}-a_{1}\right)+\left(1-\gamma_{2}\right)\left(\beta_{\mathrm{b}}+a_{2}\right) k_{1}}{\left(\gamma_{2}-\gamma_{1}\right) k_{1}^{\gamma_{1}}} \\
C_{4}=\frac{-\gamma_{1}\left(\beta_{\mathrm{s}}-a_{1}\right)+\left(\gamma_{1}-1\right)\left(\beta_{\mathrm{b}}+a_{2}\right) k_{1}}{\left(\gamma_{2}-\gamma_{1}\right) k_{1}^{\gamma_{2}}} .
\end{array}\right.
$$

Substituting these into (18), we obtain two equations on $k_{1}$ and $k_{2}$

$$
\begin{aligned}
& \frac{\gamma_{2}\left(\beta_{\mathrm{s}}-a_{1}\right)+\left(1-\gamma_{2}\right)\left(\beta_{\mathrm{b}}+a_{2}\right) k_{1}}{\left(\gamma_{2}-\gamma_{1}\right)}\left(\frac{k_{2}}{k_{1}}\right)^{\gamma_{1}} \\
& \quad+\frac{-\gamma_{1}\left(\beta_{\mathrm{s}}-a_{1}\right)+\left(\gamma_{1}-1\right)\left(\beta_{\mathrm{b}}+a_{2}\right) k_{1}}{\left(\gamma_{2}-\gamma_{1}\right)}\left(\frac{k_{2}}{k_{1}}\right)^{\gamma_{2}}+a_{1}+a_{2} k_{2} \\
& =\frac{-\delta_{4} \beta_{\mathrm{s}}+\left(\delta_{4}-1\right) \beta_{\mathrm{b}} k_{2}}{\eta_{3}\left(\delta_{3}-\delta_{4}\right)}+\frac{\delta_{3} \beta_{\mathrm{s}}+\left(1-\delta_{3}\right) \beta_{\mathrm{b}} k_{2}}{\eta_{4}\left(\delta_{3}-\delta_{4}\right)}
\end{aligned}
$$

and

$$
\begin{aligned}
& \frac{\gamma_{2}\left(\beta_{\mathrm{s}}-a_{1}\right)+\left(1-\gamma_{2}\right)\left(\beta_{\mathrm{b}}+a_{2}\right) k_{1}}{\left(\gamma_{2}-\gamma_{1}\right)} \gamma_{1}\left(\frac{k_{2}}{k_{1}}\right)^{\gamma_{1}} \\
& \quad+\frac{-\gamma_{1}\left(\beta_{\mathrm{s}}-a_{1}\right)+\left(\gamma_{1}-1\right)\left(\beta_{\mathrm{b}}+a_{2}\right) k_{1}}{\left(\gamma_{2}-\gamma_{1}\right)} \gamma_{2}\left(\frac{k_{2}}{k_{1}}\right)^{\gamma_{2}}+a_{2} k_{2} \\
& =\frac{-\delta_{4} \beta_{\mathrm{s}}+\left(\delta_{4}-1\right) \beta_{\mathrm{b}} k_{2}}{\eta_{3}\left(\delta_{3}-\delta_{4}\right)} \delta_{3}+\frac{\delta_{3} \beta_{\mathrm{s}}+\left(1-\delta_{3}\right) \beta_{\mathrm{b}} k_{2}}{\eta_{4}\left(\delta_{3}-\delta_{4}\right)} \delta_{4} .
\end{aligned}
$$

We next simplify these equations and obtain

$$
\begin{aligned}
& {\left[\gamma_{2}\left(\beta_{\mathrm{s}}-a_{1}\right)+\left(1-\gamma_{2}\right)\left(\beta_{\mathrm{b}}+a_{2}\right) k_{1}\right]\left(\frac{k_{2}}{k_{1}}\right)^{\gamma_{1}}+\gamma_{2} a_{1}+\left(\gamma_{2}-1\right) a_{2} k_{2}} \\
& \quad=\frac{-\delta_{4} \beta_{\mathrm{s}}+\left(\delta_{4}-1\right) \beta_{\mathrm{b}} k_{2}}{\eta_{3}\left(\delta_{3}-\delta_{4}\right)}\left(\gamma_{2}-\delta_{3}\right)+\frac{\delta_{3} \beta_{\mathrm{s}}+\left(1-\delta_{3}\right) \beta_{\mathrm{b}} k_{2}}{\eta_{4}\left(\delta_{3}-\delta_{4}\right)}\left(\gamma_{2}-\delta_{4}\right)
\end{aligned}
$$

and

$$
\begin{gathered}
{\left[-\gamma_{1}\left(\beta_{\mathrm{s}}-a_{1}\right)+\left(\gamma_{1}-1\right)\left(\beta_{\mathrm{b}}+a_{2}\right) k_{1}\right]\left(\frac{k_{2}}{k_{1}}\right)^{\gamma_{2}}+\left(1-\gamma_{1}\right) a_{2} k_{2}-\gamma_{1} a_{1}} \\
=\frac{-\delta_{4} \beta_{\mathrm{s}}+\left(\delta_{4}-1\right) \beta_{\mathrm{b}} k_{2}}{\eta_{3}\left(\delta_{3}-\delta_{4}\right)}\left(\delta_{3}-\gamma_{1}\right)+\frac{\delta_{3} \beta_{\mathrm{s}}+\left(1-\delta_{3}\right) \beta_{\mathrm{b}} k_{2}}{\eta_{4}\left(\delta_{3}-\delta_{4}\right)}\left(\delta_{4}-\gamma_{1}\right) .
\end{gathered}
$$


To reduce the above equations into linear equations in $k_{1}$ and $k_{2}$, we let $r=k_{2} / k_{1}$. Then, we have

$$
\begin{array}{r}
{\left[\gamma_{2}\left(\beta_{\mathrm{s}}-a_{1}\right)+\left(1-\gamma_{2}\right)\left(\beta_{\mathrm{b}}+a_{2}\right) k_{1}\right] r^{\gamma_{1}}=\left[\frac{-\delta_{4} \beta_{\mathrm{s}}\left(\gamma_{2}-\delta_{3}\right)}{\eta_{3}\left(\delta_{3}-\delta_{4}\right)}+\frac{\delta_{3} \beta_{\mathrm{s}}\left(\gamma_{2}-\delta_{4}\right)}{\eta_{4}\left(\delta_{3}-\delta_{4}\right)}-\gamma_{2} a_{1}\right]} \\
+\left[\frac{\left(\delta_{4}-1\right)\left(\gamma_{2}-\delta_{3}\right) \beta_{\mathrm{b}}}{\eta_{3}\left(\delta_{3}-\delta_{4}\right)}+\frac{\left(1-\delta_{3}\right) \beta_{\mathrm{b}}\left(\gamma_{2}-\delta_{4}\right)}{\eta_{4}\left(\delta_{3}-\delta_{4}\right)}-\left(\gamma_{2}-1\right) a_{2}\right] k_{2}
\end{array}
$$

and

$$
\begin{array}{r}
{\left[-\gamma_{1}\left(\beta_{\mathrm{s}}-a_{1}\right)+\left(\gamma_{1}-1\right)\left(\beta_{\mathrm{b}}+a_{2}\right) k_{1}\right] r^{\gamma_{2}}=\left[\frac{-\delta_{4} \beta_{\mathrm{s}}\left(\delta_{3}-\gamma_{1}\right)}{\eta_{3}\left(\delta_{3}-\delta_{4}\right)}+\frac{\delta_{3} \beta_{\mathrm{s}}\left(\delta_{4}-\gamma_{1}\right)}{\eta_{4}\left(\delta_{3}-\delta_{4}\right)}+\gamma_{1} a_{1}\right]} \\
+\left[\frac{\left(\delta_{4}-1\right)\left(\delta_{3}-\gamma_{1}\right) \beta_{\mathrm{b}}}{\eta_{3}\left(\delta_{3}-\delta_{4}\right)}+\frac{\left(1-\delta_{3}\right) \beta_{\mathrm{b}}\left(\delta_{4}-\gamma_{1}\right)}{\eta_{4}\left(\delta_{3}-\delta_{4}\right)}-\left(1-\gamma_{1}\right) a_{2}\right] k_{2} .
\end{array}
$$

To simplify notation, let

$$
\left\{\begin{array}{l}
A_{1}=\frac{-\delta_{4} \beta_{\mathrm{s}}\left(\gamma_{2}-\delta_{3}\right)}{\eta_{3}\left(\delta_{3}-\delta_{4}\right)}+\frac{\delta_{3} \beta_{\mathrm{s}}\left(\gamma_{2}-\delta_{4}\right)}{\eta_{4}\left(\delta_{3}-\delta_{4}\right)}-\gamma_{2} a_{1}, \\
A_{2}=\frac{-\delta_{4} \beta_{\mathrm{s}}\left(\delta_{3}-\gamma_{1}\right)}{\eta_{3}\left(\delta_{3}-\delta_{4}\right)}+\frac{\delta_{3} \beta_{\mathrm{s}}\left(\delta_{4}-\gamma_{1}\right)}{\eta_{4}\left(\delta_{3}-\delta_{4}\right)}+\gamma_{1} a_{1}, \\
B_{1}=\frac{\left(\delta_{4}-1\right)\left(\gamma_{2}-\delta_{3}\right) \beta_{\mathrm{b}}}{\eta_{3}\left(\delta_{3}-\delta_{4}\right)}+\frac{\left(1-\delta_{3}\right) \beta_{\mathrm{b}}\left(\gamma_{2}-\delta_{4}\right)}{\eta_{4}\left(\delta_{3}-\delta_{4}\right)}-\left(\gamma_{2}-1\right) a_{2}, \\
B_{2}=\frac{\left(\delta_{4}-1\right)\left(\delta_{3}-\gamma_{1}\right) \beta_{\mathrm{b}}}{\eta_{3}\left(\delta_{3}-\delta_{4}\right)}+\frac{\left(1-\delta_{3}\right) \beta_{\mathrm{b}}\left(\delta_{4}-\gamma_{1}\right)}{\eta_{4}\left(\delta_{3}-\delta_{4}\right)}-\left(1-\gamma_{1}\right) a_{2} .
\end{array}\right.
$$

Then, we have

$$
\left\{\begin{array}{l}
\left(\gamma_{2}\left(\beta_{\mathrm{s}}-a_{1}\right)+\left(1-\gamma_{2}\right)\left(\beta_{\mathrm{b}}+a_{2}\right) k_{1}\right) r^{\gamma_{1}}=A_{1}+B_{1} k_{2} \\
\left(-\gamma_{1}\left(\beta_{\mathrm{s}}-a_{1}\right)+\left(\gamma_{1}-1\right)\left(\beta_{\mathrm{b}}+a_{2}\right) k_{1}\right) r^{\gamma_{2}}=A_{2}+B_{2} k_{2}
\end{array}\right.
$$

Eliminate $k_{1}$ to obtain the equation in $r$ :

$$
\frac{A_{1}-\gamma_{2}\left(\beta_{\mathrm{s}}-a_{1}\right) r^{\gamma_{1}}}{\left(1-\gamma_{2}\right)\left(\beta_{\mathrm{b}}+a_{2}\right) r^{\gamma_{1}}-B_{1} r}=\frac{A_{2}+\gamma_{1}\left(\beta_{\mathrm{s}}-a_{1}\right) r^{\gamma_{2}}}{\left(\gamma_{1}-1\right)\left(\beta_{\mathrm{b}}+a_{2}\right) r^{\gamma_{2}}-B_{2} r} .
$$

Let

$$
f(r)=\frac{A_{1}-\gamma_{2}\left(\beta_{\mathrm{s}}-a_{1}\right) r^{\gamma_{1}}}{\left(1-\gamma_{2}\right)\left(\beta_{\mathrm{b}}+a_{2}\right) r^{\gamma_{1}}-B_{1} r}-\frac{A_{2}+\gamma_{1}\left(\beta_{\mathrm{s}}-a_{1}\right) r^{\gamma_{2}}}{\left(\gamma_{1}-1\right)\left(\beta_{\mathrm{b}}+a_{2}\right) r^{\gamma_{2}}-B_{2} r} .
$$

We assume

(A2) $f(r)$ has a zero $r_{0}>1$.

Use this $r_{0}$ and recall that $k_{2}=r_{0} k_{1}$ to obtain

$$
\left\{\begin{array}{l}
k_{1}=\frac{A_{1}-\gamma_{2}\left(\beta_{\mathrm{s}}-a_{1}\right) r_{0}^{\gamma_{1}}}{\left(1-\gamma_{2}\right)\left(\beta_{\mathrm{b}}+a_{2}\right) r_{0}^{\gamma_{1}}-B_{1} r_{0}}=\frac{A_{2}+\gamma_{1}\left(\beta_{\mathrm{s}}-a_{1}\right) r_{0}^{\gamma_{2}}}{\left(\gamma_{1}-1\right)\left(\beta_{\mathrm{b}}+a_{2}\right) r_{0}^{\gamma_{2}}-B_{2} r_{0}}, \\
k_{2}=r_{0} k_{1}=\frac{A_{1} r_{0}-\gamma_{2}\left(\beta_{\mathrm{s}}-a_{1}\right) r_{0}^{\gamma_{1}+1}}{\left(1-\gamma_{2}\right)\left(\beta_{\mathrm{b}}+a_{2}\right) r_{0}^{\gamma_{1}}-B_{1} r_{0}}=\frac{A_{2} r_{0}+\gamma_{1}\left(\beta_{\mathrm{s}}-a_{1}\right) r_{0}^{\gamma_{2}+1}}{\left(\gamma_{1}-1\right)\left(\beta_{\mathrm{b}}+a_{2}\right) r_{0}^{\gamma_{2}}-B_{2} r_{0}} .
\end{array}\right.
$$


Using these $k_{1}$ and $k_{2}$, we can express $C_{1}, C_{2}, C_{3}$, and $C_{4}$. Therefore, the solutions $w_{1}$ and $w_{2}$ are given by

$$
\begin{aligned}
& w_{1}(y)= \begin{cases}\beta_{\mathrm{s}}-\beta_{\mathrm{b}} y & \text { for } y \in \Gamma_{1}, \\
C_{3} y^{\gamma_{1}}+C_{4} y^{\gamma_{2}}+a_{1}+a_{2} y & \text { for } y \in \Gamma_{2}, \\
C_{1} y^{\delta_{3}}+C_{2} y^{\delta_{4}} & \text { for } y \in \Gamma_{3}\end{cases} \\
& w_{2}(y)= \begin{cases}\beta_{\mathrm{s}}-\beta_{\mathrm{b}} y & \text { for } y \in \Gamma_{1} \cup \Gamma_{2}, \\
C_{1} \eta_{3} y^{\delta_{3}}+C_{2} \eta_{4} y^{\delta_{4}} & \text { for } y \in \Gamma_{3} .\end{cases}
\end{aligned}
$$

Note that the variational inequalities in the HJB equations need to hold. In particular, we need the HJB inequalities to hold:

$$
\begin{array}{lll}
\Gamma_{1}: & \left(\rho+\lambda_{1}-\mathcal{L}_{1}\right) w_{1}(y)-\lambda_{1} w_{2}(y) \geq 0, & \left(\rho+\lambda_{2}-\mathcal{L}_{2}\right) w_{2}(y)-\lambda_{2} w_{1}(y) \geq 0 \\
\Gamma_{2}: & w_{1} \geq \beta_{\mathrm{s}}-\beta_{\mathrm{b}} y, & \left(\rho+\lambda_{2}-\mathcal{L}_{2}\right) w_{2}(y)-\lambda_{2} w_{1}(y) \geq 0 \\
\Gamma_{3}: & w_{1} \geq \beta_{\mathrm{s}}-\beta_{\mathrm{b}} y, & w_{2} \geq \beta_{\mathrm{s}}-\beta_{\mathrm{b}} y
\end{array}
$$

Next, we simplify these inequalities and establish equivalent conditions.

First, consider the inequalities on $\Gamma_{1}$. Recall that on this interval, both $w_{1}$ and $w_{2}$ equal $\beta_{\mathrm{b}}-\beta_{\mathrm{b}} y$. Simple calculation yields that

$$
\left(\rho+\lambda_{1}-\mathcal{L}_{1}\right)\left(\beta_{\mathrm{s}}-\beta_{\mathrm{b}} y\right)=\left(\rho+\lambda_{1}-\mu_{1}(1)\right) \beta_{\mathrm{s}}-\left(\rho+\lambda_{1}-\mu_{2}(1)\right) \beta_{\mathrm{b}} y .
$$

So, $\left(\rho+\lambda_{1}-\mathcal{L}_{1}\right) w_{1}(y)-\lambda_{1} w_{2}(y) \geq 0$ leads to $\left(\rho-\mu_{1}(1)\right) \beta_{\mathrm{s}}-\left(\rho-\mu_{2}(1)\right) \beta_{\mathrm{b}} y \geq 0$. This is equivalent to

$$
k_{1} \leq \frac{\left(\rho-\mu_{1}(1)\right) \beta_{\mathrm{s}}}{\left(\rho-\mu_{2}(1)\right) \beta_{\mathrm{b}}} .
$$

Similarly, if $w_{2}=\beta_{\mathrm{s}}-\beta_{\mathrm{b}} y$, then

$$
\left(\rho+\lambda_{2}-\mathcal{L}_{2}\right) w_{2}(y)=\left(\rho+\lambda_{2}-\mu_{1}(2)\right) \beta_{\mathrm{s}}-\left(\rho+\lambda_{2}-\mu_{2}(2)\right) \beta_{\mathrm{b}} y .
$$

Therefore, $\left(\rho+\lambda_{2}-\mathcal{L}_{2}\right) w_{2}(y)-\lambda_{2} w_{1}(y) \geq 0$ on $\Gamma_{1}$ is equivalent to

$$
k_{1} \leq \frac{\left(\rho-\mu_{1}(2)\right) \beta_{\mathrm{s}}}{\left(\rho-\mu_{2}(2)\right) \beta_{\mathrm{b}}} .
$$

The inequalities on $\Gamma_{1}$ are equivalent to

$$
k_{1} \leq \min \left\{\frac{\left(\rho-\mu_{1}(1)\right) \beta_{\mathrm{s}}}{\left(\rho-\mu_{2}(1)\right) \beta_{\mathrm{b}}}, \frac{\left(\rho-\mu_{1}(2)\right) \beta_{\mathrm{s}}}{\left(\rho-\mu_{2}(2)\right) \beta_{\mathrm{b}}}\right\} .
$$

Similarly, the second inequality in 21 on $\Gamma_{2}$ is equivalent to

$$
w_{1}(y) \leq \beta_{\mathrm{s}}-\beta_{\mathrm{b}} y+\frac{1}{\lambda_{2}}\left[\left(\rho-\mu_{1}(2)\right) \beta_{\mathrm{s}}-\left(\rho-\mu_{2}(2)\right) \beta_{\mathrm{b}} y\right] .
$$

To see an equivalent condition for the first inequality on $\Gamma_{2}$, let $\phi(y)=w_{1}(y)-\beta_{\mathrm{s}}+\beta_{\mathrm{b}} y$. Then $\phi\left(k_{1}\right)=0, \phi^{\prime}\left(k_{1}\right)=0$. Note that $\phi^{\prime \prime}(y)$ can have at most one zero on $\Gamma_{2}$. This implies $\phi(y) \geq 0$ on $\Gamma_{2}$ is equivalent to $\phi^{\prime \prime}\left(k_{1}\right) \geq 0$ and $\phi\left(k_{2}\right) \geq 0$. Namely,

$$
\left\{\begin{array}{l}
\phi^{\prime \prime}\left(k_{1}\right)=C_{3} \gamma_{1}\left(\gamma_{1}-1\right) k_{1}^{\gamma_{1}-2}+C_{4} \gamma_{2}\left(\gamma_{2}-1\right) k_{1}^{\gamma_{2}-2} \geq 0 \text { and } \\
\phi\left(k_{2}\right)=C_{3} k_{2}^{\gamma_{1}}+C_{4} k_{2}^{\gamma_{2}}+a_{1}+a_{2} k_{2}-\beta_{\mathrm{s}}+\beta_{\mathrm{b}} y \geq 0 .
\end{array}\right.
$$


Finally, to see an equivalent condition of the second inequality in (21) on $\Gamma_{3}$, let $\psi(y)=w_{2}(y)-\beta_{\mathrm{s}}+\beta_{\mathrm{b}} y$. Then, $\psi\left(k_{2}\right)=0, \psi^{\prime}\left(k_{2}\right)=0, \psi(\infty)=\infty$, and $\psi^{\prime}(\infty)>0$. Note also that $\psi(y)$ can have at most one zero on $\Gamma_{3}$. It follows that $\psi(y) \geq 0$ on $\Gamma_{3}$ is equivalent to $\psi^{\prime \prime}\left(k_{2}\right) \geq 0$. So, the second inequality on $\Gamma_{3}$ in 21 is equivalent to

$$
\psi^{\prime \prime}\left(k_{2}\right)=C_{1} \eta_{3} \delta_{3}\left(\delta_{3}-1\right) k_{2}^{\delta_{3}-2}+C_{2} \eta_{4} \delta_{4}\left(\delta_{4}-1\right) k_{2}^{\delta_{4}-2} \geq 0 .
$$

The other inequality on $\Gamma_{3}$ is $w_{1}(y) \geq \beta_{\mathrm{s}}-\beta_{\mathrm{b}} y$. Hence,

$$
C_{1} y^{\delta_{3}}+C_{2} y^{\delta_{4}} \geq \beta_{\mathrm{s}}-\beta_{\mathrm{b}} y .
$$

We assume these equivalent inequalities.

(A3) The inequalities in (22), (23), 24), 25), and (26) hold.

Case 2: $k_{1}=k_{2}$. In this case, let $k_{0}=k_{1}=k_{2}$. We have $w_{1}=w_{2}=\beta_{\mathrm{s}}-\beta_{\mathrm{b}} y$ on $\left(0, k_{0}\right]$ and

$$
w_{1}=C_{1} y^{\delta_{3}}+C_{2} y^{\delta_{4}} \quad \text { and } \quad w_{2}=C_{1} \eta_{3} y^{\delta_{3}}+C_{2} \eta_{4} y^{\delta_{4}}
$$

on $\left[k_{0}, \infty\right)$. Then, smooth-fit conditions imply at $k_{0}$

$$
\begin{aligned}
\beta_{\mathrm{s}}-\beta_{\mathrm{b}} k_{0} & =C_{1} k_{0}^{\delta_{3}}+C_{2} k_{0}^{\delta_{4}}, \\
-\beta_{\mathrm{b}} & =\delta_{3} C_{1} k_{0}^{\delta_{3}-1}+\delta_{4} C_{2} k_{0}^{\delta_{4}-1}, \\
\beta_{\mathrm{s}}-\beta_{\mathrm{b}} k_{0} & =\eta_{3} C_{1} k_{0}^{\delta_{3}}+\eta_{4} C_{2} k_{0}^{\delta_{4}}, \\
-\beta_{\mathrm{b}} & =\eta_{3} \delta_{3} C_{1} k_{0}^{\delta_{3}-1}+\eta_{4} \delta_{4} C_{2} k_{0}^{\delta_{4}-1} .
\end{aligned}
$$

This implies $C_{1}=C_{1} \eta_{3}$ and $C_{2}=C_{2} \eta_{4}$. Hence $w_{1}(y)=w_{2}(y)=w(y)$. Then $w(y)$ satisfies

$$
\left(\rho+\lambda_{1}-\mathcal{L}_{1}\right) w(y)=\lambda_{1} w(y) \quad \text { and } \quad\left(\rho+\lambda_{2}-\mathcal{L}_{2}\right) w(y)=\lambda_{2} w(y)
$$

for $y>k_{0}$. This yields $\left(\rho-\mathcal{L}_{1}\right) w(y)=0$ and $\left(\rho-\mathcal{L}_{2}\right) w(y)=0$. Since both $\rho-\mathcal{L}_{1}$ and $\rho-\mathcal{L}_{2}$ are of Euler type, we have $w(y)=C_{1} y^{\gamma_{0}}$ with $\gamma_{0}$ satisfying the two quadratic equations:

$$
\begin{aligned}
& \sigma_{1} \gamma_{0}\left(\gamma_{0}-1\right)+\left[\mu_{2}(1)-\mu_{1}(1)\right] \gamma_{0}+\mu_{1}(1)-\rho=0 \\
& \sigma_{2} \gamma_{0}\left(\gamma_{0}-1\right)+\left[\mu_{2}(2)-\mu_{1}(2)\right] \gamma_{0}+\mu_{1}(2)-\rho=0
\end{aligned}
$$

and taking the value:

$$
\begin{aligned}
\gamma_{0} & =\frac{1}{2}+\frac{\mu_{1}(1)-\mu_{2}(1)}{2 \sigma_{1}}-\sqrt{\left(\frac{1}{2}+\frac{\mu_{1}(1)-\mu_{2}(1)}{2 \sigma_{1}}\right)^{2}+\frac{\rho-\mu_{1}(1)}{\sigma_{1}}} \\
& =\frac{1}{2}+\frac{\mu_{1}(2)-\mu_{2}(2)}{2 \sigma_{2}}-\sqrt{\left(\frac{1}{2}+\frac{\mu_{1}(2)-\mu_{2}(2)}{2 \sigma_{2}}\right)^{2}+\frac{\rho-\mu_{1}(2)}{\sigma_{2}}} .
\end{aligned}
$$

The second equality is the necessary and sufficient condition for $k_{1}=k_{2}$. Now the smooth fitting conditions yield

$$
\beta_{\mathrm{s}}-\beta_{\mathrm{b}} k_{0}=C_{1} k_{0}^{\gamma_{0}} \text { and } \quad-\beta_{\mathrm{b}} k_{0}=C_{1} \gamma_{0} k_{0}^{\gamma_{0}} .
$$

We can solve this system and obtain

$$
k_{0}=\frac{-\gamma_{0} \beta_{\mathrm{s}}}{\left(1-\gamma_{0}\right) \beta_{\mathrm{b}}}, \quad C_{1}=\frac{-k_{0} \beta_{\mathrm{b}}}{\gamma_{0} k_{0}^{\gamma_{0}}}=\frac{\left(-\gamma_{0}\right)^{-\gamma_{0}}}{\left(1-\gamma_{0}\right)^{1-\gamma_{0}}} \cdot \frac{\beta_{\mathrm{s}}^{1-\gamma_{0}}}{\beta_{\mathrm{b}}^{-\gamma_{0}}}=\frac{\beta_{\mathrm{s}}^{1-\gamma_{0}} \beta_{\mathrm{b}}^{\gamma_{0}}}{\left(-\gamma_{0}\right)^{\gamma_{0}}\left(1-\gamma_{0}\right)^{1-\gamma_{0}}} .
$$


Note that $\gamma_{0}<0$, which implies that both $k_{0}$ and $C_{1}$ are positive. Finally the solution to the HJB equations is given by

$$
w_{1}(y)=w_{2}(y)=w(y)= \begin{cases}\beta_{\mathrm{s}}-\beta_{\mathrm{b}} y & \text { for } y \in\left(0, k_{0}\right] \\ C_{1} y^{\gamma_{0}} & \text { for } y \in\left(k_{0}, \infty\right) .\end{cases}
$$

Next we prove that the variational inequalities hold in this case. We need to show

$$
\begin{array}{ll}
\left(\rho-\mathcal{L}_{1}\right) w(y) \geq 0 \text { and }\left(\rho-\mathcal{L}_{2}\right) w(y) \geq 0 & \text { on }\left(0, k_{0}\right], \\
w(y) \geq \beta_{\mathrm{s}}-\beta_{\mathrm{b}} y & \text { on }\left(k_{0}, \infty\right) .
\end{array}
$$

We first prove the second inequality. Let $\phi_{0}(y)=w(y)-\beta_{\mathrm{s}}+\beta_{\mathrm{b}} y=C_{1} y^{\gamma_{0}}-\beta_{\mathrm{s}}+\beta_{\mathrm{b}} y$. Then, $\phi_{0}\left(k_{0}\right)=0, \phi_{0}^{\prime}\left(k_{0}\right)=0$, and $\phi_{0}^{\prime \prime}(y)>0$. It follows that $\phi_{0}(y)$ is increasing on $\left(k_{0}, \infty\right)$. Therefore, $\phi_{0}(y) \geq 0$ on this interval.

To show the first inequality in $(29)$, note that on $\left(0, k_{0}\right]$, for $i=1,2$,

$$
\left(\rho-\mathcal{L}_{i}\right) w(y)=\left(\rho-\mathcal{L}_{i}\right)\left(\beta_{\mathrm{s}}-\beta_{\mathrm{b}} y\right)=\left(\rho-\mu_{1}(i)\right) \beta_{\mathrm{s}}-\left(\rho-\mu_{2}(i)\right) \beta_{\mathrm{b}} y .
$$

Therefore,

We have

$$
\left(\rho-\mathcal{L}_{i}\right) w(y) \geq 0 \text { on }\left(0, k_{0}\right] \Longleftrightarrow k_{0} \leq \frac{\left(\rho-\mu_{1}(i)\right) \beta_{\mathrm{s}}}{\left(\rho-\mu_{2}(i)\right) \beta_{\mathrm{b}}}
$$

$$
k_{0} \leq \frac{\left(\rho-\mu_{1}(i)\right) \beta_{\mathrm{s}}}{\left(\rho-\mu_{2}(i)\right) \beta_{\mathrm{b}}} \Longleftrightarrow \frac{-\gamma_{0}}{1-\gamma_{0}} \leq \frac{\rho-\mu_{1}(i)}{\rho-\mu_{2}(i)} \Longleftrightarrow \gamma_{0}\left(\mu_{2}(i)-\mu_{1}(i)\right) \leq \rho-\mu_{1}(i) .
$$

If $\mu_{2}(i) \geq \mu_{1}(i)$, then using (27), we have $\gamma_{0} \leq 1 / 2$. It follows that

$$
\left(\mu_{2}(i)-\mu_{1}(i)\right) \gamma_{0} \leq \frac{\mu_{2}(i)-\mu_{1}(i)}{2} \leq \rho-\mu_{1}(i)
$$

because of assumption (A1). So $\left(\rho-\mathcal{L}_{i}\right) w(y) \geq 0$ on $\left(0, k_{0}\right]$ in this case.

If $\mu_{2}(i)<\mu_{1}(i)$, then we have

$$
\begin{aligned}
\gamma_{0}\left(\mu_{2}(i)-\mu_{1}(i)\right) \leq \rho-\mu_{1}(i) \\
\Longleftrightarrow\left(\mu_{2}(i)-\mu_{1}(i)\right) \sqrt{\left(\frac{1}{2}+\frac{\mu_{1}(i)-\mu_{2}(i)}{2 \sigma_{i}}\right)^{2}+\frac{\rho-\mu_{1}(i)}{\sigma_{i}}} \\
\quad \leq \rho-\mu_{1}(i)+\frac{\mu_{1}(i)-\mu_{2}(i)}{2}+\frac{\left(\mu_{1}(i)-\mu_{2}(i)\right)^{2}}{2 \sigma_{i}} \\
\Longleftrightarrow \sqrt{\left(\frac{1}{2}+\frac{\mu_{1}(i)-\mu_{2}(i)}{2 \sigma_{i}}\right)^{2}+\frac{\rho-\mu_{1}(i)}{\sigma_{i}} \leq \frac{2 \rho-\mu_{1}(i)-\mu_{2}(i)}{2\left(\mu_{1}(i)-\mu_{2}(i)\right)}+\frac{\mu_{1}(i)-\mu_{2}(i)}{2 \sigma_{i}}} \\
\Longleftrightarrow\left(\frac{1}{2}+\frac{\mu_{1}(i)-\mu_{2}(i)}{2 \sigma_{i}}\right)^{2}+\frac{\rho-\mu_{1}(i)}{\sigma_{i}} \leq\left(\frac{2 \rho-\mu_{1}(i)-\mu_{2}(i)}{2\left(\mu_{1}(i)-\mu_{2}(i)\right)}+\frac{\mu_{1}(i)-\mu_{2}(i)}{2 \sigma_{i}}\right)^{2} \\
\Longleftrightarrow \frac{\mu_{1}(i)-\mu_{2}(i)}{2 \sigma_{i}}+\frac{1}{4}+\frac{\rho-\mu_{1}(i)}{\sigma_{i}} \leq\left(\frac{2 \rho-\mu_{1}(i)-\mu_{2}(i)}{2\left(\mu_{1}(i)-\mu_{2}(i)\right)}\right)^{2}+\frac{2 \rho-\mu_{1}(i)-\mu_{2}(i)}{2 \sigma_{i}} \\
\Longleftrightarrow \frac{1}{4} \leq\left(\frac{2 \rho-\mu_{1}(i)-\mu_{2}(i)}{2\left(\mu_{1}(i)-\mu_{2}(i)\right)}\right)^{2} \Longleftrightarrow \frac{1}{2} \leq \frac{2 \rho-\mu_{1}(i)-\mu_{2}(i)}{2\left(\mu_{1}(i)-\mu_{2}(i)\right)} \Longleftrightarrow \rho \geq \mu_{1}(i),
\end{aligned}
$$

which holds due to (A1). Therefore, the inequalities in 29 hold. 
A verification theorem. We provide a verification theorem for both Cases 1 and 2 .

Theorem 3.1. In Case 1, assume (A1), (A2), and (A3). In Case 2, assume (A1). Then, $v\left(x_{1}, x_{2}, \alpha\right)=x_{1} w_{\alpha}\left(x_{2} / x_{1}\right)=V\left(x_{1}, x_{2}, \alpha\right), \alpha=1,2$. Let $D=\left\{\left(x_{1}, x_{2}, 1\right): x_{2}>k_{1} x_{1}\right\} \cup$ $\left\{\left(x_{1}, x_{2}, 2\right): x_{2}>k_{2} x_{1}\right\}$. Let $\tau^{*}=\inf \left\{t:\left(X_{t}^{1}, X_{t}^{2}, \alpha_{t}\right) \notin D\right\}$. Then $\tau^{*}$ is optimal.

Proof. The proof is similar to that of [GZ, Theorem 2]. We only sketch the main steps for the sake of completeness. First, for any stopping time $\tau$, following Dynkin's formula, we have

$$
v\left(x_{1}, x_{2}, \alpha\right) \geq E e^{-\rho \tau} v\left(X_{\tau}^{1}, X_{\tau}^{2}, \alpha_{\tau}\right) \geq E e^{-\rho \tau}\left(\beta_{\mathrm{s}} X_{\tau}^{1}-\beta_{\mathrm{b}} X_{\tau}^{2}\right)=J\left(x_{1}, x_{2}, \alpha, \tau\right) .
$$

So, $v\left(x_{1}, x_{2}, \alpha\right) \geq V\left(x_{1}, x_{2}, \alpha\right)$. The equality holds when $\tau=\tau^{*}$. Hence, $v\left(x_{1}, x_{2}, \alpha\right)=$ $J\left(x_{1}, x_{2}, \alpha, \tau^{*}\right)=V\left(x_{1}, x_{2}, \alpha\right)$.

4. Numerical examples. In this section, we give three examples, one for each case: $k_{1}<k_{2}, k_{1}=k_{2}$, or $k_{1}>k_{2}$.

EXAMPLE $4.1\left(k_{1}<k_{2}\right)$. In this example, we take

$$
\begin{array}{llll}
\mu_{1}(1)=0.20, & \mu_{2}(1)=0.25, & \mu_{1}(2)=-0.30, & \mu_{2}(2)=-0.35, \\
\sigma_{11}(1)=0.30, & \sigma_{12}(1)=0.10, & \sigma_{21}(1)=0.10, & \sigma_{22}(1)=0.35, \\
\sigma_{11}(2)=0.40, & \sigma_{12}(2)=0.20, & \sigma_{21}(2)=0.20, & \sigma_{22}(2)=0.45, \\
\lambda_{1}=6.0, & \lambda_{2}=10.0, & K=0.001, & \rho=0.50 .
\end{array}
$$

Then, we use the function $f(r)$ in 19 and find the unique zero $r_{0}=1.020254>1$. Using this $r_{0}$ and (20), we obtain $k_{1}=0.723270$ and $k_{2}=0.737920$. Then, we calculate and get $C_{1}=0.11442, C_{2}=-0.00001, C_{3}=0.29121, C_{4}=0.00029, \eta_{3}=0.985919$, and $\eta_{4}=-1.541271$. With these numbers, we verify all variational inequalities required in (A3). The graphs of the value functions are given in Figure 1
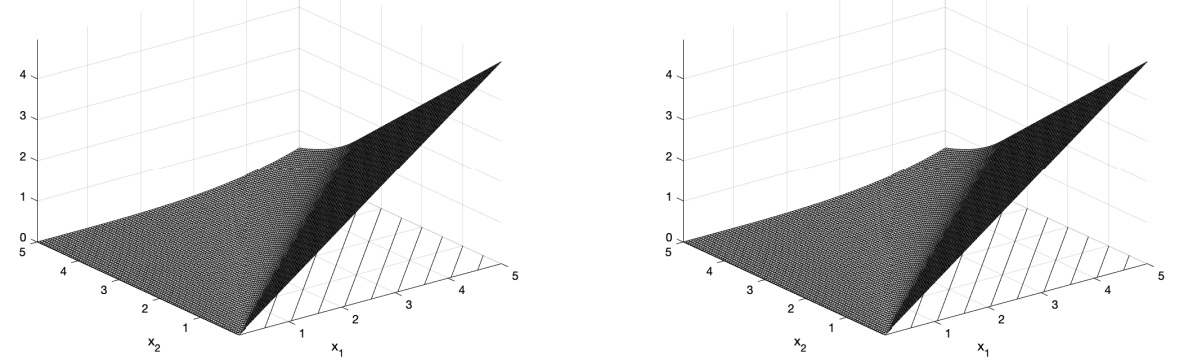

Fig. 1. Value functions $V\left(x_{1}, x_{2}, 1\right)$ and $V\left(x_{1}, x_{2}, 2\right)$

EXAMPLE $4.2\left(k_{1}=k_{2}\right)$. In this example, we take

$$
\begin{array}{ll}
\mu_{1}(1)=\mu_{1}(2)=0.20, & \mu_{2}(1)=\mu_{2}(2)=0.25, \\
\sigma_{11}(1)=\sigma_{11}(2)=0.30, & \sigma_{12}(1)=\sigma_{12}(2)=0.10, \\
\sigma_{21}(1)=\sigma_{21}(2)=0.10, & \sigma_{22}(1)=\sigma_{22}(2)=0.35, \\
\lambda_{1}=6.0, \lambda_{2}=10.0, & K=0.001, \rho=0.50 .
\end{array}
$$

Clearly, the second equality in (27) holds, which leads to $k_{1}=k_{2}=k_{0}$. Use (28) to obtain $k_{0}=0.705098$ and $C_{1}=0.126431$. This gives the corresponding value function. Its graph is given in Figure 2 


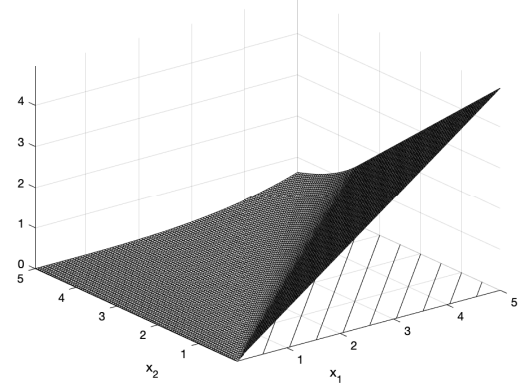

Fig. 2. Value function $V\left(x_{1}, x_{2}\right)=V\left(x_{1}, x_{2}, 1\right)=V\left(x_{1}, x_{2}, 2\right)$

EXAmPLE $4.3\left(k_{1}>k_{2}\right)$. Finally, we take a different set of parameters from those used in Example 4.1 .

$$
\begin{array}{llll}
\mu_{1}(1)=-0.10, & \mu_{2}(1)=0.20, & \mu_{1}(2)=0.25, & \mu_{2}(2)=-0.15 \\
\sigma_{11}(1)=0.35, & \sigma_{12}(1)=0.15, & \sigma_{21}(1)=0.15, & \sigma_{22}(1)=0.30 \\
\sigma_{11}(2)=0.20, & \sigma_{12}(2)=0.10, & \sigma_{21}(2)=0.10, & \sigma_{22}(2)=0.15 \\
\lambda_{1}=6.0, & \lambda_{2}=10.0, & K=0.001, & \rho=0.50
\end{array}
$$

In this example, if we apply the procedure used in Example 4.1 for $k_{1}$ and $k_{2}$, we notice some of the variational inequalities in (A3) will be violated. This means the condition $k_{1}<k_{2}$ does not apply. Based on the symmetry of the problem in $\alpha=1$ and $\alpha=2$, we switch the set of parameters about $\alpha=1$ and $\alpha=2$ and obtain $\tilde{k}_{1}=0.379300$ and $\tilde{k}_{2}=0.824070$. The 'new' value functions $\left(\tilde{V}\left(x_{1}, x_{2}, 1\right), \tilde{V}\left(x_{1}, x_{2}, 2\right)\right)$ can be obtained in a similar way. So are the verification of the variational inequalities in (A3). Then, we switch back to obtain $k_{1}=\tilde{k}_{2}=0.824070$ and $k_{2}=\tilde{k}_{1}=0.379300$. The same for the value functions $\left(V\left(x_{1}, x_{2}, 1\right)=\tilde{V}\left(x_{1}, x_{2}, 2\right)\right.$ and $\left.V\left(x_{1}, x_{2}, 2\right)=\tilde{V}\left(x_{1}, x_{2}, 1\right)\right)$. Their graphs are given in Figure 3 .
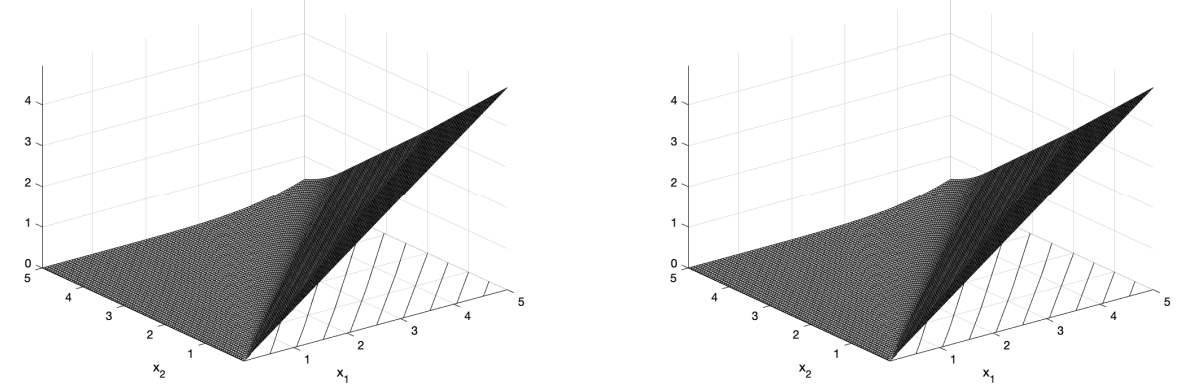

Fig. 3. Value functions $V\left(x_{1}, x_{2}, 1\right)$ and $V\left(x_{1}, x_{2}, 2\right)$

5. Conclusions. The main focus of this paper is on a pairs trade selling rule. It extends the results of McDonald and Siegel [MS and $\mathrm{Hu}$ and $\varnothing \mathrm{ksendal}[\mathrm{HO}$ ] by incorporating models with regime switching. It would be interesting to extend the results to include the buying side of optimal timing. Besides, it would also be interesting to consider models in which the market mode $\alpha_{t}$ is not directly observable. In this case, the Wonham filter can be used for calculation of the conditional probabilities of $\alpha=1$ given the stock prices up 
to time $t$. Some ideas along this line have been used in Dai et al. DZZ] in connection with trend following trading.

\section{References}

[DZZ] M. Dai, Q. Zhang, Q. Zhu, Trend following trading under a regime switching model, SIAM J. Financial Math. 1 (2010), 780-810.

[DB] A. Deshpande, B. R. Barmish, A general framework for pairs trading with a controltheoretic point of view, in: 2016 IEEE Conference on Control Applications (CCA), Buenos Aires, Argentina, September 19-22, 2016.

[EHM] R. J. Elliott, J. van der Hoek, W. P. Malcolm, Pairs trading, Quant. Finance 5 (2005), $271-276$.

[GGR] E. Gatev, W. N. Goetzmann, K. G. Rouwenhorst, Pairs trading: Performance of a relative-value arbitrage rule, Rev. Financ. Stud. 19 (2006), 797-827.

[GZ] X. Guo, Q. Zhang, Optimal selling rules in a regime switching model, IEEE Trans. Automat. Control 50 (2005), 1450-1455.

[HO] Y. Hu, B. Øksendal, Optimal time to invest when the price processes are geometric Brownian motions, Finance Stoch.2 (1998), 295-310.

[IB] S. Iwarere, B. R. Barmish, A confidence interval triggering method for stock trading via feedback control, in: 2010 American Control Conference (Baltimore, MD, 2010), IEEE, 2010, 6910-6916.

[MS] R. McDonald, D. Siegel, The value of waiting to invest, Quart. J. Econom. 101 (1986), 707-727.

[MZ] A. Merhi, M. Zervos, A model for reversible investment capacity expansion, SIAM J. Control Optim. 46 (2007), 839-876.

[O] B. Øksendal, Stochastic Differential Equations, 6th ed., Springer, Berlin, 2003.

[SZ] Q. S. Song, Q. Zhang, An optimal pairs-trading rule, Automatica J. IFAC 49 (2013), 3007-3014.

[TZZ] J. Tie, H. Zhang, Q. Zhang, An Optimal strategy for pairs-trading under geometric Brownian motions, J. Optim. Theory Appl. 179 (2018), 654-675.

[W] M. Whistler, Trading Pairs: Capturing Profits and Hedging Risk with Statistical Arbitrage Strategies, Wiley, Hobokon, 2004.

[ZZ] H. Zhang, Q. Zhang, Trading a mean-reverting asset: Buy low and sell high, Automatica J. IFAC 44 (2008), 1511-1518.

[Z] Q. Zhang, Stock trading: An optimal selling rule, SIAM J. Control Optim. 40 (2001), 64-87. 